\title{
Educação Permanente em Saúde na Estratégia de Saúde da Família: Relato da Elaboração do Plano de Intervenção
}

\author{
Permanent Health Education in the Family Health Strategy: Report on the Preparation of \\ the Intervention Plan
}

\begin{abstract}
Educación permanente en salud en la estrategia de salud familiar: Informe sobre la preparación del plan de intervención
\end{abstract}

Michelle Ingrid Assis da Silva ${ }^{1 \star}$, Jessica de Alencar Nascimento ${ }^{1}$, Juliana Conceição Dias Garcez ${ }^{1}$, Nádile Juliane Costa de Castro², Shirley Aviz de Miranda'.

\section{RESUMO}

Objetivo: Relatar a experiência sobre o processo de elaboração de um plano de intervenção relacionado à Educação Permanente em Saúde na Estratégia de Saúde da Família. Relato da Experiência: Relato de experiência de abordagem qualitativa, realizado entre 2017-2018 em uma Estratégia de Saúde da Família em Belém do Pará. A coleta de dados foi composta por roda de conversa com 12 participantes e utilizou-se o Planejamento Estratégico Situacional, como metodologia para a construção do Plano de intervenção. Não houve necessidade de aprovação em comitê de ética e pesquisa, contudo os aspectos éticos foram respeitados. O problema priorizado foi a "Baixa efetividade da política de Educação Permanente em Saúde na Estratégia", embasado nele construiu-se o plano interventivo que apresenta os objetivos, estratégias, público alvo e resultados esperados. Observou-se que a maioria das propostas criadas para o plano tem viabilidade. Considerações finais: A criação do Plano foi facilitada graças à metodologia escolhida que auxiliou na escolha da problemática, referente à educação permanente em saúde pela equipe, além de facilitar a identificação de sua importância para a eficiência dos serviços de saúde prestados, caracterizando uma maior probabilidade de sucesso e eficácia na aplicabilidade futura do plano de intervenção e redução da problemática priorizada.

Palavras-chave: Planejamento Estratégico, Estratégia de Saúde da Família, Atenção Primária em Saúde, Educação Permanente.

\begin{abstract}
Objective: To report the experience on the process of elaborating an intervention plan related to Permanent Health Education in the Family Health Strategy. Experience Report: Report of experience of qualitative approach, conducted between 2017-2018 in a Family Health Strategy in Belém do Pará. Data collection consisted of a conversation circle with 12 participants and the Strategic Situational Planning was used. as a methodology for the construction of the intervention plan. There was no need for approval by the ethics and research committee, however the ethical aspects were respected. The priority problem was the "Low effectiveness of the Permanent Health Education policy in the Strategy", based on it, the intervention plan that presents the objectives, strategies, target audience and expected results. Most of the proposals created for the plan were found to be viable. Final considerations: The creation of the Plan was facilitated thanks to the chosen methodology that helped in the choice of the problem related to permanent health education by the team, besides facilitating the identification of its importance for the efficiency of the health services provided, characterizing a higher probability. of success and effectiveness in the future applicability of the intervention plan and reduction of the prioritized problem.
\end{abstract}

Key words: Strategic Planning, Family Health Strategy, Primary Health Care, Continuing Education.

${ }^{1}$ Centro Universitário Metropolitano da Amazônia (Unifamaz), Belém-Pará. *E-mail: michelleingrid.as@gmail.com

2 Universidade Federal do Pará (UFPA), Belém-Pará. 


\section{RESUMEN}

Objetivo: Informar sobre la experiencia en el proceso de elaboración de un plan de intervención relacionado con la Educación Permanente en Salud en la Estrategia de Salud Familiar. Informe de experiencia: Informe de experiencia de enfoque cualitativo, realizado entre 2017 y 2018 en una Estrategia de salud familiar en Belém do Pará. La recopilación de datos consistió en un círculo de conversación con 12 participantes y se utilizó la Planificación situacional estratégica. como metodología para la construcción del plan de intervención. No hubo necesidad de aprobación por parte del comité de ética e investigación, sin embargo, se respetaron los aspectos éticos. El problema prioritario fue la "Baja efectividad de la política de Educación Permanente en Salud en la Estrategia", basada en ella, el plan de intervención que presenta los objetivos, estrategias, público objetivo y resultados esperados. La mayoría de las propuestas creadas para el plan resultaron ser viables. Consideraciones finales: La creación del Plan se facilitó gracias a la metodología elegida que ayudó en la elección del problema relacionado con la educación permanente en salud por parte del equipo, además de facilitar la identificación de su importancia para la eficiencia de los servicios de salud prestados, caracterizando una mayor probabilidad. de éxito y efectividad en la aplicabilidad futura del plan de intervención y la reducción del problema priorizado.

Palabras clave: Planificación Estratégica, Estrategia de Salud de la Familia, Atención Primaria en Salud, Educación Permanente.

\section{INTRODUÇÃO}

O processo de solidificação da Reforma Sanitária Brasileira que culminou na Lei orgânica da Saúde, reconheceu a formação profissional como um dos fatores imprescindíveis para a atuação em saúde no país, constituindo-o como relevante para a criação, anos depois, da Política Nacional de Educação Permanente em Saúde (PNEPS), que objetiva promover mudanças nas práticas do trabalho com base no pensamento crítico-reflexivo de seus atores (CARDOSO MLM, et. al., 2017; LEMOS CLS, 2016).

A denominação Educação Permanente em Saúde (EPS), citada na década de 80 pela Organização Pan Americana de Saúde (OPAS), ampliou o conceito antes atribuído a educação continuada, onde a EPS passa a promover um maior diálogo entre o aprender e o ensinar na realidade dos serviços em saúde, no qual o trabalhador tem maior envolvimento em seu processo produtivo da saúde, acarretando na necessidade de um olhar mais atencioso para as questões relacionadas a ela (BRASIL, 2017a; LEMOS CLS, 2016; PORTELA GZ, 2017).

Nestes termos, abordar a EPS no contexto da Atenção Primária em Saúde (APS) direciona-se pela a Estratégia Saúde da Família (ESF), a qual se constitui como estratégia prioritária para expansão, reorganização, qualificação e consolidação da APS. É uma política que contribui para qualificação profissional por meio de um fazer reflexivo e de qualidade do serviço prestado, pois suscita reflexões e modificações importantes nos processos de trabalho, situações de saúde das pessoas e coletividades, tornando um solo fértil para a utilização da EPS (BRASIL, 2017b).

Cabe ressaltar que desenvolver pesquisas voltadas para EPS na ESF é sumariamente importância, pois permite contribuir com a comunidade científica por meio de um olhar mais direcionado para a importância da qualidade dos serviços de saúde e da educação em serviço, fortalecendo a utilização da EPS como uma estratégia mais abrangente para o favorecimento dos processos de trabalho em saúde (CARDOSO MLM, et. al., 2017; LEMOS CLS, 2016).

Nesse contexto, o objetivo deste artigo é relatar a experiência sobre o processo de elaboração de um plano de intervenção relacionado à Educação Permanente em Saúde na Estratégia de Saúde da Família no município de Belém/PA.

\section{RELATO DE EXPERIÊNCIA}

Trata-se de um relato de experiência, com abordagem qualitativa, realizados no período entre 2017-2018 em Belém do Pará, como resultado das vivências obtidas durante as atividades teóricas-práticas do curso de 
Pós-graduação em Atenção Primária com ênfase em Saúde da Família do Centro Universitário Metropolitano da Amazônia (Unifamaz).

O local da pesquisa foi uma ESF localizada em Belém do Pará, composta por duas equipes, a equipe 1 formada por seis Agentes Comunitário da Saúde (ACS), um Enfermeiro, um Médico e um Técnico em Enfermagem e a equipe 2 composta por oito ACS; um Enfermeiro, um médico e um Técnico em enfermagem.

Como critério de inclusão, todos os participantes devem ser profissionais da ESF em atividade no período de execução da experiência. Para desenvolvimento da experiência foram realizadas rodas de conversa composta por 9 profissionais que compõem as equipes, além das pesquisadoras, buscando conhecer a realidade e vivências da ESF e de seus processos de trabalho, permitindo assim o levantamento das problemáticas existentes, identificando pontos positivos e negativos da atuação na ESF.

Não foi necessária aprovação em comitê de ética (CEP) e pesquisa, assim como a assinatura do Termo de Consentimento Livre e Esclarecido (TCLE), devido às atividades de levantamento de problemas terem ocorrido apenas por meio de rodas de conversa, ficando dispensado protocolo de apresentação do CEP.

A partir das falas dos participantes emergiram quatro problemas vinculados, principalmente as fragilidades na Educação Permanente em Saúde (EPS), dificuldades na comunicação entre equipe e gestão, e na adesão da comunidade aos tratamentos sugeridos, além de desvalorização profissional. Essa variedade de demandas relatadas demonstrou a necessidade de intervenções que visem priorizar problemas mais emergenciais, a fim de gradativamente diminui-las ou até mesmo eliminá-las, para assim ampliar a qualidade dos serviços de saúde da ESF. As rodas de conversas possibilitaram coleta de dados inicial, que de acordo com as quatro etapas do Planejamento Estratégico Situacional (PES), configura-se como momento Explicativo, onde realizamos a observação da realidade e levantamento de problemas como observado por Genlini JA (2014). As demais etapas, Momento Normativo; Momento Estratégico, e Momento Táticooperacional, foram desenvolvidas ao longo da elaboração do plano de intervenção (PI) (GENLINI JA, 2014).

Com base nessas etapas e no cenário apresentado e observado durante as visitas, realizou-se a priorização dos problemas por meio da Matriz de Priorização de Problemas (MPP), (Tabela 1), e posteriormente, construiu-se a Árvore de Problemas (AP), (Figura 1), ambas referentes aos momentos Explicativo e Normativo, que correspondem, respectivamente, primeira e segunda etapa do PES.

Tabela 1 - Matriz de Priorização de Problemas, n=12. Belém-PA, 2018.

\begin{tabular}{|c|c|c|c|c|c|}
\hline Problema & Magnitude & Transcendência & Vulnerabilidade & Factibilidade & $\begin{array}{l}\text { Ordem de } \\
\text { priorização }\end{array}$ \\
\hline $\begin{array}{l}\text { Fragilidade na } \\
\text { educação } \\
\text { permanente }\end{array}$ & $\begin{array}{r}+++ \\
\text { (Alta) } \\
\text { № } 19\end{array}$ & $\begin{array}{c}++ \\
\text { (Média) } \\
\text { № } 18\end{array}$ & $\begin{array}{c}++ \\
\text { (Média) } \\
\text { № } 16\end{array}$ & $\begin{array}{c}+++ \\
\text { (Alta) } \\
\text { № } 16\end{array}$ & $\begin{array}{c}1^{\circ} \\
№ 69\left(^{*}\right)\end{array}$ \\
\hline $\begin{array}{l}\text { Fragilidade na } \\
\text { comunicação } \\
\text { entre } \\
\text { profissionais e } \\
\text { gestores }\end{array}$ & $\begin{array}{l}++++ \\
\text { (Muito Alta) } \\
\text { № } 22\end{array}$ & $\begin{array}{l}+++ \\
\text { (Alta) } \\
\text { № } 12\end{array}$ & $\begin{array}{c}++ \\
\text { (Média) } \\
\text { № } 13\end{array}$ & $\begin{array}{c}+++ \\
\text { (Alta) } \\
\text { № } 10\end{array}$ & $\begin{array}{c}3^{\circ} \\
\text { № } 57\end{array}$ \\
\hline $\begin{array}{l}\text { Desvalorização } \\
\text { da equipe em } \\
\text { relação a gestão }\end{array}$ & $\begin{array}{l}++++ \\
\text { (Muito Alta) } \\
\text { № } 18\end{array}$ & $\begin{array}{c}++ \\
\text { (Média) } \\
\text { № } 11\end{array}$ & $\begin{array}{c}+++ \\
\text { (Alta) } \\
\text { № } 12\end{array}$ & $\begin{array}{c}++ \\
\text { (Média) } \\
\text { № } 12\end{array}$ & $\begin{array}{c}4^{\circ} \\
\text { № } 53\end{array}$ \\
\hline $\begin{array}{l}\text { Baixa adesão } \\
\text { dos tratamentos } \\
\text { na ESF }\end{array}$ & $\begin{array}{l}++++ \\
\text { (Muito Alta) } \\
\text { № } 19\end{array}$ & $\begin{array}{c}+++ \\
\text { (Alta) } \\
\text { № } 15 \\
\end{array}$ & $\begin{array}{c}++ \\
\text { (Média) } \\
\text { № } 15\end{array}$ & $\begin{array}{c}+++ \\
\text { (Alta) } \\
\text { № } 13\end{array}$ & $\begin{array}{c}2^{\circ} \\
\text { № } 62\end{array}$ \\
\hline
\end{tabular}

Legenda: Grau De Importância: Baixa [+]; Média [++]; Alta [+++]; Muito Alta [++++]; №: Refere-se à quantidade de pontuação TOTAL atribuída por cada ator envolvido em cada um dos tópicos e problemas elencado; $\left({ }^{*}\right)$ : Refere-se ao problema que será priorizado por se apresentar o mais relevante e viável dos demais problemas elencados na somatória dos pontos.

Fonte: Silva MIS, Nascimento JA, Garcez JCD, et al., 2018. 
Pode-se observar que compuseram a matriz de priorização, quatro problemas principais, resultantes da roda de conversa e segundo a percepção subjetiva de cada participante envolvido (Tabela 1). Esses problemas foram avaliados considerando os critérios objetivos de decisão: Magnitude; Transcendência; Vulnerabilidade e Factibilidade, atribuindo-se Notas e Graus de Importância, representados aqui por cruzes e pontos, por razão direta, ou seja, quanto maior importância atribuída a cada problema maior a pontuação e o grau de importância aplicado (TEIXEIRA CF, et al., 2010).

Logo, o tema gerador/ problema passou a ser a "Baixa efetividade da política de Educação Permanente em Saúde na ESF". A partir de então foi iniciada a segunda etapa, Momento Normativo, com a construção da $A P$, que buscou definir as causas/nós críticos, descritores e consequências, (Figura 1).

Figura 1 - Organograma da Árvore de Problemas, Belém-PA, 2018.

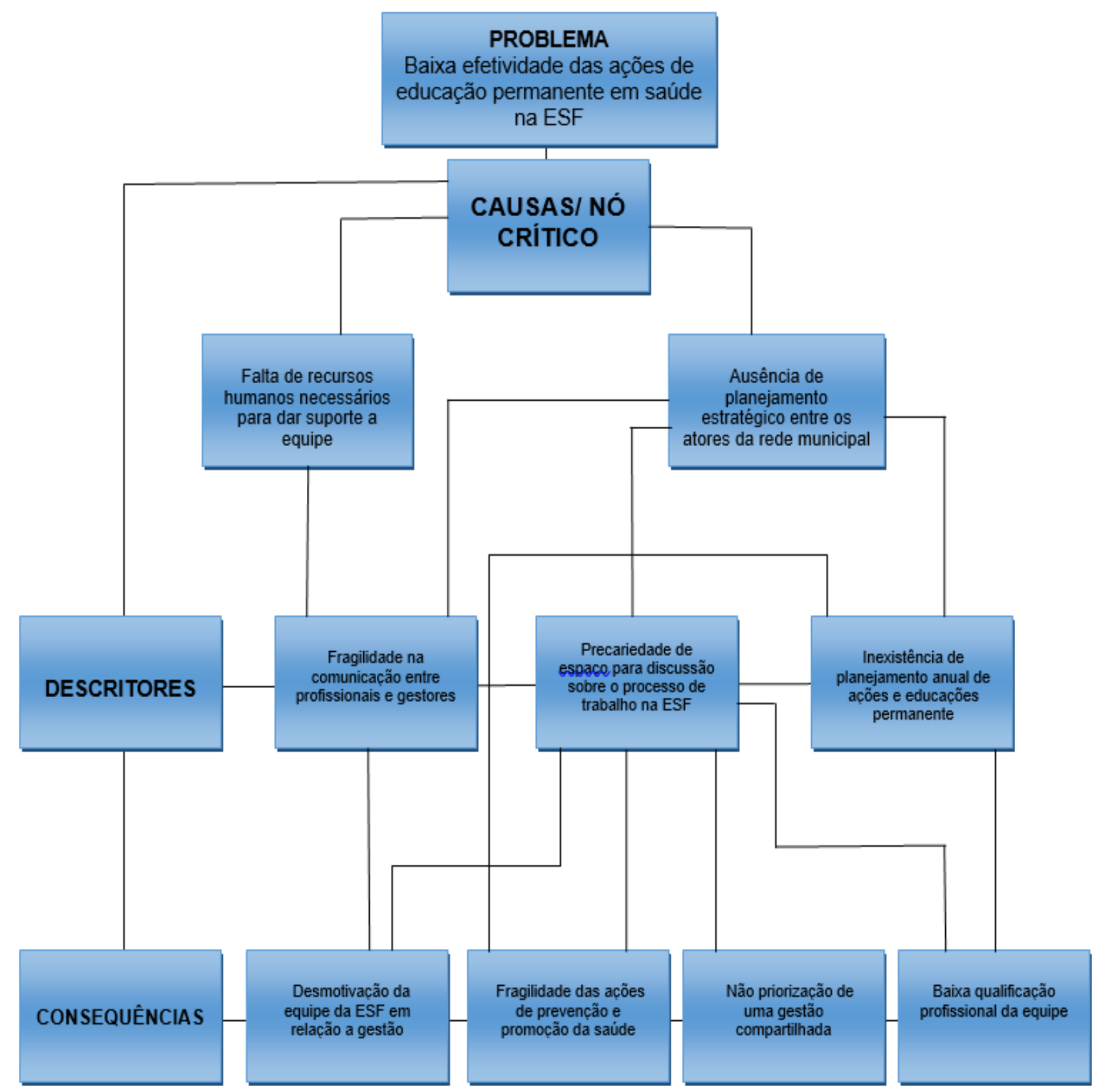

Fonte: Silva MIS, Nascimento JA, Garcez JCD, et al., 2018. 
Oribe CY (2012), diz que a AP ou explicativa é uma ferramenta simples, de fácil utilização, que apresenta vantagens em relação a outras metodologias, principalmente pelo fato de se adequar aos diversos ambientes, contextos e áreas de atuação, além do melhor desempenho no processo de identificação da causa/nós críticos e consequências do problema, tornando-o essencial para que esses sejam anteriormente priorizados e caso não sejam resolvidos, sejam ao menos minimizados (SOUZA BBC, 2010).

Após a realização da somatória dos pontos atribuídos em cada problema, a "Fragilidade na Educação Permanente" foi a problemática que se apresentou mais prioritária e viável para ser alvo de intervenção no cenário de pesquisa. Contudo, foi realizada uma adaptação do problema, somente para proporcionar uma melhor compreensão do mesmo, e sem que houvesse alteração do sentido atribuído inicialmente.

As causas/nós-críticos da problemática elencada pelos atores envolvidos foram: 1) - falta de recursos humanos necessários para dar suporte a equipe; e 2) - ausência de planejamento estratégico entre os atores da rede municipal.

A causa/ nó crítico 1, acarretou como consequência a "desmotivação da equipe da ESF em relação a gestão" e como descritor a "fragilidade na comunicação entre profissionais e gestores".

Já a causa/ nó crítico 2, abarcou todas as relações entre os descritores e consequências gerados. Os três descritores para esse nó foram a "Fragilidade na comunicação entre profissionais e gestores"; "Inexistência de planejamento anual de ações e educações permanente" e "Precariedade de espaço para discussão sobre o processo de trabalho na ESF", e estão ligados diretamente a ele.

A consequência para o primeiro descritor, assim como foi na causa 1, continua sendo a "desmotivação da equipe da ESF em relação a gestão", já o segundo descritor para a causa 2, gerou como consequências a "fragilidade das ações de prevenção e promoção da saúde"; e "baixa qualificação profissional da equipe". O terceiro descritor que gerou, também, todas as consequências citadas até aqui, acrescentou ainda a "não priorização de uma gestão compartilhada".

O fato da causa/ nó crítico 2, referente a ausência de planejamento estratégico, ter gerado todos os descritores e consequências, comprova-nos, mais uma vez, a relevância do planejamento para a manutenção e qualidade das relações de trabalho e o serviço de saúde. Além disto, pode-se verificar que a construção de um planejamento, realizado de forma descentralizada e coletiva entre os atores envolvidos e a rede é sumariamente necessária para a efetivação da melhoria na qualidade dos serviços prestados e efetivação das metas estabelecidas, pois, todos envolvidos, passam a reconhecer e visualizar as melhorias advindas do planejamento no seu cotidiano.

Neste sentido tanto a MPP quanto a AP foram ferramentas essenciais para que um problema prioritário, suas causas e consequências fossem encontradas, a fim de direcionar os objetivos que nortearam a construção do PI. Além disso, fica clara a necessidade e relevância da participação ativa dos atores envolvidos nesse processo, buscando obter demandas advindas das vivencias reais de cada indivíduo, pois são esses que estão diretamente no local da intervenção e dos processos de trabalho.

Sendo assim, após as primeiras etapas e por meio dos nós críticos mencionados, chegou-se a terceira etapa do PES, momento estratégico, que remete a construção do Plano de Intervenção (PI), que segundo Schneider DR e Flach PMV (2014) é uma proposta de ação objetiva que visa promover mudanças numa dada realidade partindo da identificação de problemas e necessidades, a fim de resolvê-las, tendo como base a Metodologia da Problematização (MP) por meio do Planejamento Estratégico Situacional (PES), pois é uma ferramenta que permite explorar o direcionamento das ações, para alcance dos resultados desejados, focando nos problemas existentes de uma realidade, sobre a qual se pretende agir e/ou transformar (KLEBA ME, et al., 2011).

O PI terá ações voltadas para EPS a serem desenvolvidas posteriormente na ESF alvo do estudo (Tabela 2). Ressalta-se que Silva KL, et al. (2017), citam que se pode utilizar denominações como "treinamento", "capacitação", para referir-se a ações traçadas para a EPS. 
Tabela 2 - Plano interventivo em Educação Permanente em Saúde. Belém-PA, 2018.

\begin{tabular}{|c|c|c|}
\hline \multicolumn{3}{|c|}{ PROBLEMA: Baixa efetividade das ações de educação permanente na ESF } \\
\hline Nós Críticos & $\begin{array}{l}\text { Nó crítico 1: Falta de recursos } \\
\text { humanos para dar suporte a equipe. }\end{array}$ & $\begin{array}{l}\text { Nó crítico 2: Ausência de planejamento } \\
\text { estratégico entre os atores da rede } \\
\text { municipal }\end{array}$ \\
\hline Estratégias & $\begin{array}{l}\text { Oficinas e Palestras direcionadas } \\
\text { conforme a PNEPS (2017). } \\
\text { Qualificação dos profissionais } \\
\text { Distribuições de tarefas para } \\
\text { otimizar o serviço. } \\
\text { Reuniões frequentes entre gestão e } \\
\text { equipe para tratar sobre os } \\
\text { trabalhos desenvolvidos. } \\
\text { Realização de concurso público. } \\
\text { Processos seletivos para } \\
\text { contratação de pessoal. }\end{array}$ & $\begin{array}{l}\text { Oficinas e Palestras voltadas para a } \\
\text { EPS } \\
\text { Planejamento anual } \\
\text { Relatórios semanais } \\
\text { Gestão compartilhada } \\
\text { Motivação da equipe } \\
\text { Promoção da discussão sobre os } \\
\text { processos de trabalho por meio de } \\
\text { reuniões de equipe semanais }\end{array}$ \\
\hline Objetivos & $\begin{array}{l}\text { Capacitar a equipe multidisciplinar, } \\
\text { a fim de motivar e fortalecer o } \\
\text { processo de trabalho desenvolvido, } \\
\text { bem como elucidar a importância do } \\
\text { trabalho em equipe e ampliação do } \\
\text { quadro profissional. }\end{array}$ & $\begin{array}{l}\text { Capacitar a equipe da ESF para } \\
\text { realização de planejamento estratégico } \\
\text { contínuo e compartilhado }\end{array}$ \\
\hline Períodos & Conforme a necessidade. & Anual \\
\hline Público Alvo & $\begin{array}{l}\text { Gestores e equipe de profissionais } \\
\text { da ESF. }\end{array}$ & $\begin{array}{l}\text { Atores envolvidos da ESF e Gestores } \\
\text { municipais. }\end{array}$ \\
\hline $\begin{array}{l}\text { Resultados } \\
\text { Esperados }\end{array}$ & $\begin{array}{l}\text { Profissionais preparados para } \\
\text { atender com qualidade e presteza a } \\
\text { população contribuindo para } \\
\text { concretização do sistema único de } \\
\text { saúde. }\end{array}$ & $\begin{array}{l}\text { Desenvolvimento de ações planejadas } \\
\text { periodicamente e promoção da } \\
\text { melhoria na comunicação e articulação } \\
\text { entre equipe e gestão. }\end{array}$ \\
\hline
\end{tabular}

Fonte: Silva MIS, Nascimento JA, Garcez JCD, et al., 2018

No que se refere a viabilidade para a aplicabilidade do PI, verificou-se que as propostas de ações apresentadas são viáveis para intervenção, pois considera-se que é apenas necessário o envolvimento e comprometimento dos atores envolvidos para a realização e concretização do plano.

No entanto, os dois últimos itens do nó crítico 1, "Realização de concurso público" e "Processos seletivos para contratação de pessoal", apresentam as viabilidades um pouco comprometidas a curto e médio prazo, pois para que aconteçam são necessários, além do comprometimento dos atores envolvidos, meios e recursos não só humanos mais financeiros e governamentais para que sejam efetivados, logo ficam apenas como proposta de ação futura para a gestão.

Por fim para a quarta etapa, momento tático-operacional, ressalta-se que em virtude da concepção e objetivo desse artigo, não se objetivou a aplicabilidade do $\mathrm{PI}$, e sim relatar a experiência para a construção do mesmo. Logo essa etapa não será descrita nessa pesquisa.

Portanto, a metodologia empregada ofereceu um dinamismo e embasamento para a construção do PI por direcionar a priorização dos problemas de forma detalhada, sem deixar de ser clara.

Contudo, por terem métodos extensos, são necessários um pouco mais de tempo para que as etapas sejam devidamente seguidas e finalizadas. 


\section{RESULTADOS E DISCUSSÃO}

A escolha do método de (PES), permitiu identificar a maneira mais adequada de lidar com a complexidade da realidade social e das situações que acabam surpreendendo o gestor e a equipe (NASCIMENTO JO, REIS MPR, 2015).

A primeira etapa do PES é o Momento Explicativo que busca identificar, definir, analisar e priorizar os problemas provenientes da realidade dos atores envolvidos. Nesta etapa, é possível questionar e compreender os problemas enfrentados seu teores e possíveis causas (CAZAGRANDE GS e PONTES AT, 2018; ARTMANN E, 2012; GENTILINI JA, 2014).

Na segunda etapa, Momento Normativo, define-se os nós críticos e as causas dos problemas identificados, considerando sempre a realidade dos atores envolvidos, a fim de dar procedência aos objetivos e ações que serão realizados (CAZAGRANDE GS e PONTES AT, 2018; VEBER AP et al., 2011).

A terceira etapa, Momento Estratégico, é a analisa da viabilidade do plano político-econômico e organizacional, entre o que se almeja e o que é possível (CAZAGRANDE GS e PONTES AT, 2018; GENTILINI JA, 2014).

Por fim a última etapa, Momento Tático-operacional, é o momento de gerenciar e monitorar a execução das ações do plano inserido (CAZAGRANDE GS e PONTES AT, 2018; ALVES AF et. al., 2008; VEBER AP et. al., 2011; ARTMANN E, 2012).

A partir da problemática priorizada ficou evidente a fragilidade quanto a educação permanente na ESF, daí a importância do PI baseado na EPS, pois esta deve ser tomada como um recurso estratégico e permanente que, através da sua efetivação em saúde, otimiza políticas, prioriza a organização da rede de atenção, e propõem transformações significativas nas práticas profissionais (BRASIL, 2017a).

A própria PNEPS ressalta a necessidade de que o trabalhador participe de práticas educativas, sejam elas em forma de palestras, cursos ou outros eventos dessa natureza, cuja finalidade seja capacitar os profissionais de saúde, bem como instrumentalizá-los para enfrentar os problemas do seu dia a dia de trabalho, deixando clara a importância estratégica da EPS para potencializar e fortalecer os trabalhadores e usuários para novas práticas de assistência, ensino, gestão e participação social (SILVA KL, et al., 2017; BRASIL, 2017a).

Silva KL, et al. (2017), diz que esses profissionais passam a desenvolverem uma escuta mais aguçada, capaz de entender as questões trazidas pelos usuários e estabelecerem com eles uma comunicação mais eficaz. Ademais, construir um PI foi desafiador, pois diversos contextos, socio-político-econômico e operacionais, devem ser considerados para que as ações futuras sejam, não apenas viáveis, mas também eficazes.

Além disso, sensibilizar os profissionais quanto à relevância desse processo de priorização, construção, e torná-los atores ativos, são um dos maiores desafios, pois grande parte dos discursos emergem desanimo e descrença quanto às possíveis mudanças no serviço e sua real efetivação. Logo, para o alcance dos objetivos e melhorias dos serviços de saúde, essa apropriação por parte dos profissionais em relação ao seu trabalho é necessária.

\section{CONSIDERAÇÕES FINAIS}

Conclui-se que a experiência na ESF propiciou a integração das pesquisadoras com os profissionais do local, permitindo vários olhares e impressões significativas que auxiliaram na problematização da realidade. Do mesmo modo, o PES, aliado a MPP e a AP, direcionaram a trajetória metodológica da criação do PI, ligando a prática assistencial de uma equipe multidisciplinar em saúde e a pesquisa científica, bem como, possibilitou o diálogo entre atores envolvidos, tornando-os agentes ativos na construção do PI e de seu fazer ocupacional, partindo sempre das concepções subjetivas de cada um, a fim de alcançar um consenso coletivo da equipe sobre o que deve ser priorizado. A EPS atribuiu a essa equipe a identificação de sua importância para a eficiência dos serviços de saúde prestados, caracterizando assim uma maior probabilidade de sucesso 
e eficácia na aplicabilidade futura do PI construído, no entanto, ainda são necessários mais estudos que fomentem a temática, a fim de trazer mais dados e embasamentos para a construção de PI, por meio do PES, e relacionados a EPS, especificamente na ESF, que é modelo da APS e núcleo direcionador das redes de atenção no país.

\section{REFERÊNCIAS}

1. ALVES AF, et. al. (2008), "Planejamento Estratégico como ferramenta de gestão para organização e integração da Assistência Farmacêutica na Atenção Primária a Saúde", Congresso Virtual Brasileiro. 15 p. Disponível em: http://www.convibra.com.br/upload/paper/2013/61/2013_61_7791.pdf. Acesso em: 15 dez. 2018.

2. ARTMANN E. (2012), "O planejamento estratégico situacional no nível local: um instrumento a favor da visão multissetorial”. Núcleo de Educação em Saúde Coletiva, Universidade Federal de Minas Gerais. 25 p. Disponível em: https://www.nescon.medicina.ufmg.br/biblioteca/imagem/2153.pdf. Acesso em: 20 jan. 2019.

3. BAUER MW, et al. Qualidade quantidade e interesse do conhecimento: evitando confusões. in BAUER MW., GASKELL G. e org(s). 13.ed. Pesquisa qualitativa com texto, imagem e som: um manual prático. Petrópolis, Rio De Janeiro: Vozes. 2017; 520 p.

4. BRASIL. Ministério da Saúde. Conselho Nacional de Saúde. Resolução n. 466, de 12 de dezembro de 2012. Aprova diretrizes e normas regulamentadoras de pesquisas envolvendo seres humanos. Brasília, Diário Oficial da União, 2012; 12 p.

5. BRASIL. Conselho Nacional de Secretários de Saúde. A Gestão do SUS. Brasília: CONASS, $2015 ; 133$ p.

6. BRASIL. Ministério da Saúde. Gabinete do Ministro. Portaria N 3.194, De 28 De Novembro De 2017. Dispõe sobre o Programa para o Fortalecimento das Práticas de Educação Permanente em Saúde no Sistema Único de Saúde PRO EPS-SUS. Brasília, DF: Biblioteca Virtual em Saúde, 2017a; 6 p.

7. BRASIL. Ministério da Saúde. Portaria no 2.436, de 21 de setembro de 2017. Aprova a Política Nacional de Atenção Básica, estabelecendo a revisão de diretrizes para a organização da Atenção Básica, no âmbito do Sistema Único de Saúde (SUS). Brasília, DF: Biblioteca Virtual em Saúde, 2017b; 32 p.

8. CARDOSO MLM, et al. A Política Nacional de Educação Permanente em Saúde nas Escolas de Saúde Pública: reflexões a partir da prática. Ciência\& Saúde Coletiva, 2017; 22(5): 1489-1500.

9. CAZAGRANDE GS, PONTES AT. Aplicação Do Planejamento Estratégico Situacional Na Central De Abastecimento Farmacêutico De Um Hospital Público De Médio Porte. Sistemas \& Gestão, 2018; 13: 25-35.

10. GENTILINI JA. Atores, cenários e planos: O planejamento estratégico situacional e a educação. Cadernos de pesquisa, 2014; 44(153): 580-601.

11. KLEBA ME, et al. O Planejamento Estratégico Situacional no Ensino da Gestão em Saúde da Família. Contexto Enferm, Florianópolis, 2011; 20(1): 184-93.

12. LEMOS CLS. Educação Permanente em saúde no Brasil: educação ou gerenciamento permanente?. Ciência \& Saúde Coletiva, 2016; 21(3): 913-922.

13. NASCIMENTO JO, REIS MPR. Planejamento Estratégico Situacional. Revista Liceu On-line, São Paulo, 2015; 5(1): 86-101.

14. ORIBE CY. Diagrama de Árvore: a ferramenta para os tempos atuais. Qualypro, 2012; 5.

15. SCHNEIDER DR, FLACH PMV. Caderno de orientações para o projeto de intervenção: curso prevenção dos problemas relacionados ao uso de drogas: capacitação para conselheiros e lideranças comunitárias. SENADMJ/NUTE-UFSC, Brasília - DF, 2014; 60 p.

16. SILVA KL, et al. A construção da educação permanente no processo de trabalho em saúde no estado de Minas Gerais, Brasil. Escola Anna Nery, 2017; 21(4): 8.

17. SORATTO J, et al. Estratégia Saúde Da Família: Uma Inovação Tecnológica Em Saúde. Contexto Enferm, Florianópolis, 2015; 24(2): 584-92.

18. SOUZA BCC. Gestão da mudança e da inovação: árvore de problemas como ferramenta para avaliação do impacto da mudança. Revista de Ciências Gerenciais, São Paulo, 2010; 14(19): 18.

19. TEIXEIRA CF, et al. Proposta Metodológica para o planejamento no Sistema Único De Saúde. In TEIXEIRA CF. (org.). Planejamento em saúde: conceitos, métodos e experiências. Salvador: EDUFBA, 2010; $161 \mathrm{p}$.

20. VEBER AP, et al. Planejamento em saúde - Unidade 2. In VEBER AP. et al. Gestão da assistência farmacêutica, Módulo Transversal 1. Universidade Federal de Santa Catarina, Universidade Aberta do SUS, Florianópolis: UFSC, $2011 ; 149 \mathrm{p}$.

21. PORTELA GZ. Atenção Primária à Saúde: um ensaio sobre conceitos aplicados aos estudos nacionais. Physis Revista de Saúde Coletiva, Rio de Janeiro, 2017; 27(2): 255-276. 